\title{
Aprender inglés en la escuela desde los tres años
}

\author{
InMa Piquer Vives \\ Universidad de Barcelona \\ Enviado: 10 Marzo 2006 / Aceptado 24 Abril 2006 \\ ISSN: $1697-7467$
}

\begin{abstract}
RESUMEN. Avanzar la introducción del inglés al parvulario es un objetivo que responde a una fuerte demanda social que no queda libre de dificultades ni de riesgos de fracaso. A pesar de todo, este artículo nos demuestra que, desde las escuelas, es posible conseguir buenos resultados sin caer en propuestas didácticas complicadas ni gastos extraordinarios.
\end{abstract}

Palabras clave: introducción del inglés, parvulario, propuesta didáctica.

\begin{abstract}
English Language Teaching to very young learners is the answer to a very strong social pressure that isn't free from difficulties or risk of failure. However, this article demonstrates that it is possible to achieve successful results from schools without having to follow complicated and expensive proposals.

Keywords: English language teaching, very young learners, proposals.
\end{abstract}

\section{INTRODUCCIÓN}

El objetivo principal que persigue la enseñanza de la lengua inglesa en las escuelas de Educación Infantil del país es el de sensibilizar a los alumnos de forma progresiva hacia el aprendizaje de la lengua y cultura anglosajonas. La buena calidad del primer contacto con la lengua garantizará a los alumnos un acercamiento progresivo que repercutirá de forma positiva en sus aprendizajes futuros. Por este motivo es cada vez más frecuente encontrar centros que deciden poner en práctica proyectos dirigidos a avanzar la introducción del inglés en la Educación Infantil.

A pesar del optimismo y la ilusión con que muchas escuelas se acogen a esta iniciativa, no siempre resulta factible desarrollar los proyectos en toda su magnitud. En demasiadas ocasiones existen diferentes factores que dificultan la introducción del inglés en el parvulario. Algunos de estos factores son: las presiones sociales, las limitaciones económicas que se traducen en una falta significativa de recursos humanos, espaciales y materiales en las escuelas; un fondo insuficiente de medios dirigidos a la formación de profesionales especializados en la enseñanza de la LE en la Educación Infantil; una oferta limitada de propuestas funcionales y rigurosas que puedan garantizar resultados satisfactorios en las aulas, etc.

El objetivo de este artículo es el de mostrar las diferentes etapas seguidas en el diseño de una propuesta dirigida a avanzar la introducción del inglés en la Educación Infantil en una escuela pública de Cataluña. La propuesta que se presenta a continuación se caracteriza por: ser económicamente viable, ofrecer unos resultados de calidad y posibilitar su aplicación en 
cualquier escuela pública del país con las modificaciones pertinentes. Esto implica partir de unas bases metodológicas claras y bien definidas que servirán de guía durante todo el proceso de diseño, aplicación y evaluación de la propuesta didáctica.

La naturaleza de la iniciativa llevada a cabo en las aulas del parvulario del CEIP Sant Cristòfor de Begues: avanzar la introducción del inglés en el parvulario, ha exigido una metodología diversificada. En primer lugar, la metodología propia de la investigación teórica, en segundo lugar, una investigación curricular; en tercer lugar, una investigación empírica que, dada la naturaleza de la investigación, se adecua a las bases que marca la investigaciónacción. Esta opción metodológica se ha considerado la más apropiada para el pilotaje y evaluación de la acción didáctica. Por tanto, las tres vertientes metodológicas de la investigación son:

- Investigación teórica. Se realiza a partir de la revisión minuciosa e intensa de la bibliografía relacionada con el tema objeto de estudio.

- Investigación curricular. Se desarrolla desde una doble vertiente. Por una parte, se trata de hacer una revisión intensa y cuidadosa de las disposiciones legales en curso con la finalidad de definir una nueva propuesta curricular. Una actividad esta muy significativa y laboriosa ya que implica la elaboración y el diseño de una propuesta curricular inexistente. Esta labor en cuestión exige hacer una revisión minuciosa de los documentos de curso oficial y de uso generalizado, así como también los de curso institucional y de uso interno: el Proyecto Curricular de Centro, el Proyecto Lingüístico y las Programaciones de aula. Por otro lado, exigió el diseño de la propuesta didáctica llevada a cabo en el CEIP Sant Cristòfor haciendo un buen uso de las dotes de creatividad e inventiva de la profesional responsable.

- Investigación empírica: Investigación-acción. Se trata de un proceso planificado de acción, observación, reflexión y evaluación, de carácter cíclico conducido y negociado por los agentes implicados, con el propósito de intervenir en su práctica educativa para mejorarla.

Cohen y Manion (1990) proponen un proceso de investigación-acción integrado por ocho etapas bien diferenciadas:

1. Comprender, identificar, formular y evaluar un problema que se percibe como crítico en una situación de enseñanza-aprendizaje.

2. Consensuar el diseño de la acción didáctica a realizar en el centro.

3. Hacer una revisión minuciosa de la bibliografía referente a la enseñanza de lengua extranjeras a alumnos muy jóvenes.

4. Redefinir el problema inicial y formular los objetivos generales y específicos de la propuesta que se prevé diseñar.

5. Seleccionar de forma cuidadosa los procedimientos a seguir en la aplicación de la propuesta didáctica.

6. Elegir los instrumentos de recogida de datos que se prevé utilizar.

7. Tras la elaboración la propuesta didáctica se procede a su aplicación.

8. Interpretación de los datos y discusión de los resultados. 
Superadas las primeras etapas del proceso, las dirigidas a la documentación y el diseño de la propuesta didáctica, fue necesario pensar en los procedimientos a seguir en su aplicación y las fuentes de recogida de datos que se utilizarían en el proceso de evaluación. El uso de fuentes de recogida de datos permitió al profesional docente disponer de una información que, una vez triangulada, facilitaría la evaluación y posterior revisión de la propuesta diseñada.

Existen formas diversas de observar el proceso de adquisición de la lengua extranjera que sigue el alumno de preescolar (Pedrós, 1994):

1. Observando la forma que tiene el alumno de moverse en situaciones de comunicación y registrándolo por escrito.

2. Tomando nota de los comentarios hechos por los padres con respecto a las muchas transferencias que el alumno hace en casa.

3. A través del registro de observaciones diarias.

4. Mediante filmaciones de algunas de las sesiones.

Concretando la forma como se desarrolló el proceso de recogida de información a lo largo de la investigación llevada a cabo, acontece hacer una breve descripción de los instrumentos de recogida de datos utilizados. El primero de ellos es de carácter subjetivo y los otros dos son objetivos.

- El diario: Es el documento que sirve de testimonio puntual de las experiencias y observaciones recogidas durante el proceso de aplicación de la propuesta. Este documento ha de recoger el seguimiento de la acción, las incidencias que han tenido lugar y la valoración personal que refleja las sensaciones y preocupaciones de la persona.

- Los cuestionarios: Este instrumento permite captar información de las familias en lo que respecta al funcionamiento de la aplicación práctica de la propuesta. El valor de esta información es indiscutible, sobre todo si tenemos en cuenta que las respuestas recogidas en los cuestionarios se fundamentan en la experiencia práctica que tienen las familias del aprendizaje del inglés que hacen sus hijos en la escuela.

- Filmaciones en video: Este instrumento permite obtener información objetiva sobre los resultados de la acción.

A continuación se muestra de forma detallada la información relacionada con el proceso de investigación llevado a cabo en las aulas del parvulario del CEIP Sant Cristòfor de Begues durante el curso académico 2002-2003.

\section{Contexto de la investigación}

La propuesta diseñada para promover el avance en la introducción del inglés a las aulas de educación infantil fue aplicada y evaluada con posterioridad en el CEIP Sant Cristófor de Begues durante el curso académico 2002-2003.

Se trata de una escuela pequeña, de una sola línea, que desde hace seis cursos ha ido creciendo de forma ininterrumpida. El alumnado de la escuela se puede considerar, en conjunto, 
poco conflictivo, aunque resulta habitual encontrar alumnos con algunos problemas de hábitos. En los últimos años, se ha apreciado un aumento progresivo en el número de alumnos que desconocen la lengua vehicular, el catalán, y en ocasiones también la L1, el castellano.

El equipo de profesionales del centro comparte la idea que la escuela ha de ofrecer recursos como: la organización del parvulario por rincones, el avance en la introducción del inglés desde el primer curso de la Educación Infantil, la organización de grupos y de actividades de aprendizaje por talleres, una oferta amplia y actualizada de recursos informáticos al alcance de todos los alumnos, todo tipo de apoyos materiales y humanos que puedan ayudar a los alumnos más desfavorecidos por lo que a la adquisición de los aprendizajes respecta, etc., ya que se considera necesaria la ampliación de la oferta de formación desde la escuela.

Los padres han demostrado un gran interés para que en la escuela se avance la introducción del inglés ya que lo consideran fundamental para la educación de sus hijos. La valoración muy positiva del avance de la lengua inglesa a $2^{\circ}$ de Primaria durante los cursos 1999 2000 y 2000-2001 hecha por el claustro fue uno de los factores más decisivos a la hora de votar a favor de la introducción del inglés en el parvulario. La iniciativa pionera de introducir el inglés al $2^{\circ}$ curso de ciclo inicial se desarrolló en el centro en el marco de la modalidad del Proyecto Orator que promueve el avance de la lengua inglesa al ciclo inicial de Primaria. El éxito del programa ha hecho que la iniciativa se haya mantenido desde entonces y que los profesionales del centro, haciéndose eco de las insistentes demandas realizadas por los padres y madres de los alumnos, hayan valorado de forma positiva la posibilidad de iniciar la introducción del inglés en el parvulario (Català, 2001).

\section{Consideraciones previas}

Se recomienda que la propuesta diseñada por el centro asegure los siguientes aspectos para garantizar así la calidad del avance: asegurar su continuidad, atender a unos principios de temporización coherentes, velar por un tratamiento conjunto de las lenguas según el Proyecto Lingüístico y la evaluación interna del área de lengua, desarrollar el currículum del área en el marco de Proyecto Curricular y velar por la formación del profesorado.

\subsection{Garantía de continuidad de la propuesta presentada}

El hecho de que el aprendizaje de la lengua extranjera se distribuya a lo largo de un periodo mayor (Educación Infantil y Primaria) permitirá efectuar una mejor distribución y dosificación de las diversas etapas del aprendizaje, así como también programar una enseñanza de más alto nivel en los grados superiores. Sin embargo, para que esta mejora cualitativa a lo largo de las diferentes etapas educativas se manifieste en el conocimiento lingüístico de los alumnos debe existir garantía de continuidad. Cualquier iniciativa de avance de la introducción de la LE ha de contar con el apoyo de todo el claustro de profesores, la aprobación del Consejo Escolar y el compromiso de todos para garantizar así que aquel avance tenga continuidad a lo largo del tiempo.

Desgraciadamente, con más frecuencia de la deseable, los centros docentes chocan con inconvenientes tales como: el cambio constante de la plantilla debido a la asignación de 
vacantes; el poco apoyo económico, material y humano que facilitan las entidades responsables; la falta de tiempo y formación para programar las clases de LE.

Estos inconvenientes se convierten en iniciativas que quedan truncadas en algún momento del proceso educativo convirtiéndose en propuestas aisladas que, pese a la repercusión positiva que tienen en los alumnos, no tienen mayor trascendencia en el futuro.

\subsection{Temporización}

Existe acuerdo unánime por lo que respecta a la convicción de que la eficacia del aprendizaje conseguido por los alumnos aumenta cuando los alumnos son sometidos a un número considerable de horas de exposición a la lengua objeto de aprendizaje (Nussbaum, 1999). Es decir, cuando los aprendices reciben un input cuantitativamente importante (Muñoz y Nussbaum, 1997). De aquí la importancia que tiene velar por la posibilidad de aumentar, en la medida de lo posible, la cantidad de horas de instrucción en lengua inglesa recibida por los alumnos.

\subsection{Tratamiento conjunto de las lenguas según el Proyecto Lingüístico y la evaluación interna del área de lengua}

El dominio de dos lenguas extranjeras además de la/s propia/s se convierte en un derecho de los ciudadanos de la nueva Europa. Garantizar una enseñanza de lenguas que, además de favorecer un tratamiento conjunto de las mismas, promueva la comunicación en diferentes lenguas en la clase ha de ser el objetivo principal del Proyecto Lingüístico del centro.

En un centro como el CEIP Sant Cristòfor resulta relativamente fácil garantizar un tratamiento conjunto de las dos lenguas de uso habitual: el catalán y el castellano. El reto aparece cuando hay que introducir una tercera que los alumnos desconocen y que difícilmente podrán utilizar fuera del contexto escolar. Delante de esta situación, la maestra se ve forzada a buscar oportunidades para interactuar con los alumnos a partir de una metodología basada en la enseñanza de la lengua oral por andamiaje; esto significa seguir de forma aproximada la siguiente estrategia general (Navalón, 1996):

- Atender las intenciones y producciones de los alumnos.

- Acoger y valorar sus producciones.

- Comprobar que se ha comprendido bien el significado de la producción.

- Incluir cada producción del alumno en la siguiente producción del profesor.

- Animar al alumno a que vaya ampliando el significado de sus propias producciones.

- Continuar estas interacciones de forma progresiva en un proceso continuo de construcción y negociación conjunta del uso del lenguaje.

\subsection{Desarrollo curricular del área en el marco del proyecto curricular}

Una programación de actividades de lengua extranjera diseñada a través de su uso como lengua vehicular de otra materia del currículum o de contenidos transversales ofrece muchas garantías de éxito (Caballero y Masats, 1999). Sin embargo, una programación de estas características no puede realizarse a ciegas, hay que recoger las prescripciones básicas establecidas por la ley y contemplarlas en el diseño de la propuesta: 
- Velar para que los contenidos de la lengua extranjera estén contextualizados en la experiencia de los alumnos.

- Promover el principio de atención individualizada.

- Asegurar la relación con otras áreas del currículum.

\subsection{Formación del profesor de inglés de Educación Infantil}

Además de tener una formación especializada en la enseñanza infantil, dominar la LE, tener una visión de conjunto sobre las características fundamentales de la organización y la estructura del discurso y sobre el funcionamiento de la lengua, conocer la metodología para la enseñanza de lenguas, ser consciente del esfuerzo y trabajo que supone dar continuidad a un proyecto de avance en la introducción de la LE, etc., el especialista de inglés en este periodo educativo ha de poder observar, registrar y analizar las interacciones verbales y no verbales que se producen en la clase, es decir, su propio comportamiento y el de sus alumnos.

Se busca, en definitiva, al maestro de inglés de Educación Infantil que, por encima de ser un hablante competente en lengua inglesa, es un comunicador dinámico y seguro, así como también una persona capaz de adentrarse con toda comodidad en el mundo de la educación infantil para conseguir su objetivo principal: comunicarse eficazmente en inglés con sus interlocutores de preescolar.

La primera condición, “sine qua non", para comunicarnos eficazmente con otras personas es establecer un buen contacto, sintonizar con el interlocutor, establecer una visión del mundo compartida, negociada, que, aunque sea parcial, nos permita referirnos a las mismas realidades y usar las mismas palabras de un modo significativo (Cantero y de Arriba, 1997:72).

Partiendo de esta premisa, una propuesta de calidad dirigida al avance del inglés en la Educación Infantil tiene muchas garantías de resultar exitosa en cualquier contexto educativo. Sin embargo, hay que añadir que siempre se recomienda hacer una revisión, adecuación y ampliación (si se considera necesario) de las actividades ofertadas en función de las necesidades y características del centro, el profesor y el alumnado.

\section{Disposiciones CURriculares y PRopuesta didáctica}

La elaboración de una propuesta curricular dirigida a la introducción del inglés en la Educación Infantil es una labor que requiere de una investigación minuciosa en el campo de las disposiciones curriculares vigentes, así como también de un ejercicio creativo bien fundamentado en la experiencia profesional. Se trata, al fin y al cabo, de diseñar un documento inexistente teniendo en cuenta los siguientes factores:

- Las exigencias establecidas en el "Diseño Curricular Base" por lo que respecta al logro de ciertos objetivos al finalizar una etapa educativa. Prestar atención a las prescripciones curriculares expuestas en el "Currículum de Educación Infantil", en el "Currículum de Educación Primaria" y en el documento "La llengua estrangera al cicle inicial. Aspectes organitzatius y didàctics".

- Introducir la enseñanza del inglés en Educación Infantil bajo una perspectiva comunicativa y significativa para los alumnos.

- El Proyecto Curricular de Centro. 
El hecho de que el currículum de Educación Infantil se caracterice por su flexibilidad resulta ideal para introducir un nuevo elemento innovador como lo es la introducción de la lengua extranjera.

La distribución temporal y horaria es uno de los factores a concretar de forma minuciosa desde un principio, ya que será el horario establecido quien nos permita elaborar el tercer nivel de concreción con realismo y coherencia. La confección de este horario debe realizarse teniendo en cuenta las necesidades del centro, la disponibilidad horaria del maestro o maestra de inglés pero, sobretodo, teniendo en cuenta la necesidad de proporcionar a los alumnos una formación en lengua inglesa frecuente. Sin embargo, no debemos fijar toda nuestra atención en este factor, existen otros tales como: la planificación, la elección de una metodología de trabajo y la relación temática entre áreas que no pueden llevarse a cabo de forma aleatoria.

La quantitat d'hores de contacte amb la llengua meta, si bé és molt important, no és l'únic aspecte que cal tenir en compte. En aquesta primera etapa és indispensable que hi hagi una bona planificació en el treball de totes les llengües d'aprenentatge, un mateix tipus de metodologia, així com un cert lligam entre els temes que es treballen en altres àrees (Generalitat de Catalunya, 2004:9).

La experiencia y el sentido común nos dicen que han de ser los grupos de P-3 los más mimados a la hora de confeccionar el horario del curso. Reservar un espacio diario; de corta duración; de dinámica repetitiva y rutinaria; y de gran interés para los alumnos por tratarse de un tiempo dirigido a los cuentos, las canciones, las marionetas, etc., se considera apropiado para la instrucción en lengua inglesa a los alumnos de P-3.

Por lo que respecta a los grupos de P-4 y P-5 se ha pensado en una distribución horaria por talleres. Esta distribución del alumnado permite trabajar con grupos reducidos sin hacer un gasto extraordinario ni de personal ni de actividades de aprendizaje ya que la misma programación semanal sirve para ambos grupos.

\subsection{Propuesta didáctica de P-3: Sesiones cortas}

Estas sesiones tienen frecuencia diaria y una duración de treinta minutos por sesión. Una buena opción de ubicación de las sesiones en la jornada escolar es dentro de la hora que sucede a la llegada del patio de los alumnos por la mañana. El porqué de esta elección se debe a dos motivos principales. Por un lado, ubicando las sesiones de inglés justo después del patio aseguramos que los alumnos estén receptivos a actividades memorísticas ya que vienen del recreo. Por otro lado, respetamos las actividades prioritarias del P-3 que los alumnos han de realizar con el tutor o tutora del grupo, las rutinas que se realizan a primera y última hora de la mañana o de la tarde (saludo, poner batas, sentarse en la alfombra, pasar lista, decir el día de la semana, mirar el tiempo, hablar sobre el fin de semana, comentar el menú del día, despedirse, etc.).

La programación de estas sesiones se hace tomando la semana como núcleo temático, se realizan programaciones semanales sin tener en cuenta la correspondencia con la distribución mensual. El contenido de las sesiones es muy flexible.

Un modelo de sesión podría ser aquel en el que el maestro o maestra de inglés llega al aula con un saco o un carrito lleno de elementos con los que los alumnos ya están familiarizados. Es optativo que el maestro acompañe su llegada con música aunque un acompañamiento musical siempre dará más solemnidad a la llegada y a la despedida. 
Con su llegada al aula, no cabe duda de que el adulto habrá captado la atención de los alumnos que irán demostrando interés por conocer el contenido del carrito. Conviene que, en todo momento, el adulto se muestre receptivo a las demandas hechas por los alumnos. Por ejemplo, si piden que enseñe la marioneta-mascota, lo tendrá que hacer para no decepcionarlos ni coartar el proceso comunicativo.

Algunas de las actividades fijas en cualquier sesión son: presentación de la mascota, aprendizaje o repaso de alguna canción, visionado de flashcards con la finalidad de aprender o repasar vocabulario, explicación de una historia a través de un cuento o un Big Book y despedida mediante la mascota. Partiendo de esta base, podemos ir añadiendo o sustituyendo alguna de estas actividades por otras diferentes: visionado de algún vídeo, ejecución de algún baile o danza, representación de alguna historia mediante marionetas o Power Point, etc.

Los alumnos de P-3 manifiestan gran interés hacia las actividades de aprendizaje que giran alrededor de la literatura infantil, las canciones, las danzas y, en general, folklore infantil, el mundo de la representación teatral, las marionetas, etc., así es que estos elementos tendrán que ser protagonistas indispensables de la programación. Puntualmente y de forma constante, conviene repetir sesiones e incluso programaciones semanales a lo largo del curso. Estas repeticiones periódicas facilitarán el elemento de rutina que permitirá a los alumnos conocer las actividades y participar activamente en el desarrollo de la sesión.

\subsection{Propuesta didáctica de P-4 y P-5: Talleres en inglés}

El planteamiento de las sesiones en inglés con los alumnos de estos cursos se ha hecho por bloques temáticos de duración variable.

Los bloques temáticos a trabajar se distribuyen estableciendo una serie de sesiones de las cuales la primera va dirigida a: presentación del tema, introducción del vocabulario correspondiente, explicación de la consistencia de todo el bloque temático y realización de alguna actividad plástica. Las sesiones restantes van dirigidas a la consolidación de los aprendizajes adquiridos a través de la primera sesión y de otras anteriores y a la confección de un mural.

Una concepción de la plástica que vaya más allá de la mera actividad manipulativa, más centrada en el reconocimiento, la identificación y la creación de formas de expresión que tuvieran en cuenta el color, las formas, las texturas, la composición... resultaría más enriquecedora para aprender inglés y para el aprendizaje estético (Pérez \& Roig, 2004:117).

La preparación de la primera sesión del bloque temático exige el esfuerzo del adulto por decorar la clase con referentes y estímulos visuales que le permitan hacer la presentación del tema delante de los alumnos sin tener de recurrir al uso de la L1. Esta presentación servirá para ubicar a los alumnos en el contexto en el que tendrán que trabajar durante las próximas sesiones del bloque temático. Una vez finalizados los prolegómenos, conviene aprovechar la primera sesión para iniciar a los alumnos en la realización del trabajo artístico que tendrán que continuar haciendo durante las sesiones posteriores.

Las siguientes sesiones del bloque temático se iniciarán con un repaso fugaz del vocabulario presentado durante la primera sesión para continuar con la labor de educación visual y plástica propiamente dicha. Un recurso a tener en cuenta durante el desarrollo de estas sesiones para favorecer la comunicación en inglés en el aula es el de montar una tienda en un rincón de la clase donde poder vender y comprar el material. Los alumnos se comunican 
en inglés en el proceso de intercambio de material en la tienda a la vez que disfrutan del aspecto lúdico de este rincón de juego simbólico.

Se recomienda el uso de música ambiental (canciones en inglés aprendidas y no aprendidas en clase) mientras se desarrollan las actividades de las sesiones dirigidas a la confección del mural. Por lo que respecta a la explicación de historia, también conviene pensar en ellas como en un recurso que, puntualmente y siempre que el tiempo nos lo permita, nos sirva para iniciar, complementar o concluir alguna de estas sesiones.

El adulto, por su parte, será el responsable de garantizar un entorno de trabajo lleno de estímulos visuales relacionados con el tema trabajado que faciliten la comunicación en inglés en el aula (imágenes representativas del nuevo vocabulario, elementos en 3D igualmente relacionados con el tema que se trabaja, mural que inspire el trabajo plástico de los alumnos, pósters y referentes que ayuden a recordar el vocabulario de uso habitual en el aula, participación puntual en el aula de otros adultos que también hablen en inglés, etc.). El uso de estímulos disuasorios (música ambiental de canciones inglesas) y estimulantes (lengua inglesa como herramienta de comunicación en la clase) que garantizan, en la medida de lo posible, una situación de inmersión lingüística en el aula.

\section{RECOGIDA Y ANÁlisis DE DATOS}

Siguiendo las prescripciones que marca la investigación-acción, una vez finalizado el diseño de propuesta didáctica y antes de proceder a su aplicación, se procede a seleccionar las fuentes de recogida de datos que permitirán hacer el análisis posterior. En el caso que nos ocupa, se han considerado: el cuestionario, el diario y el registro en video los instrumentos más idóneos en el proceso de recogida de datos.

\subsection{Los cuestionarios}

El uso de los cuestionarios ha venido motivado por el interés en conocer la opinión de las familias por lo que respecta al avance en la introducción del inglés en el parvulario.

Con respecto a los índices de participación, cabe decir que las familias más decididas a la hora de hacer saber su opinión sobre la experiencia fueron las de los alumnos de P-3 con un $80 \%$ de participación. Las de P-4 lo hicieron en un 61'40\% y las de P-5 sólo en un 28'5\%. En resumen, el $57^{\prime} 89 \%$ del total de las familias de los alumnos del parvulario hicieron saber su opinión a través de los cuestionarios.

Entre las respuestas dadas hay algunas que dan información sobre el perfil lingüístico de los alumnos y las hay que dan información sobre la valoración de la experiencia hecha por las familias. Son precisamente estas segundas las que más valor tienen a la hora de hacer la triangulación de los datos. Esto es debido a que reflejan de forma fiel la opinión de las familias sobre la propuesta y el conocimiento que tienen del nivel lingüístico de los alumnos a partir de las transferencias hechas en casa.

El uso de las escalas de actitudes ayuda al investigador a determinar la fuerza de la actitud u opinión de la persona que responde sobre diversas aseveraciones de actitudes. Asignando un código numérico a cada elemento en la escala, se puede determinar un compuesto 
o escala que representa la variedad de actitudes dentro del grupo estudiado. El propósito es poner a las personas que responden en la necesidad de estudiar sus actitudes y sentimientos sobre varias cuestiones (McKernan, 1996:143).

Cabe destacar la gran aprobación de la experiencia manifestada por los padres de los alumnos de P-3 quienes, con un $20 \%$ de respuestas muy positivas hacia el funcionamiento de la introducción del inglés en el parvulario, son el grupo de familias consultadas que con más entusiasmo han acogido esta propuesta. En segundo lugar destaca el 12\% de respuestas muy satisfactorias hacia la experiencia dadas por las familias del grupo de alumnos de P-5 y por último se hará mención del $6 \%$ dado por las familias de los alumnos de P-4.

Esta gradación por lo que respecta a la opinión de las familias recogida a través de los cuestionarios está muy estrechamente relacionada con las apreciaciones recogidas por la maestra en el diario de clase. En él destacan de forma muy significativa las mejoras conseguidas por los alumnos de P-3 por lo que respecta al conocimiento y uso de la lengua en el aula, la espontaneidad y soltura de los alumnos de P-5 a la hora de comunicarse en inglés durante el desarrollo de las sesiones de talleres y las limitaciones y reservas que tienen para hacerlo los de P-4.

\subsection{El diario}

El diario de clase ha sido el instrumento de recogida de datos utilizado por la maestra con la finalidad de poder llevar un registro anecdótico de los datos relacionados con el día a día de la acción en las aulas.

\subsubsection{Incidencias con los alumnos}

El alumnado, en general, ha respondido de forma muy entusiasta a la propuesta de introducir el inglés de forma lúdica y muy interactiva. Las canciones, los cuentos, videos, marionetas, confección de murales, etc., han sido elementos fundamentales en el desarrollo de las sesiones que, tal y como se esperaba, han supuesto el éxito de la propuesta.

Los alumnos más decididos no tardaron mucho en repetir y reproducir las canciones y expresiones que la maestra utilizaba para comunicarse con ellos. Sin embargo, algunos alumnos, sobre todo los de los grupos de P-4, manifestaron algunas dificultades a la hora de comunicarse en inglés en el aula. Los registros realizados por la maestra en su diario muestran una cierta desilusión respecto al planteamiento de las sesiones por talleres y a la limitación productiva de algunos alumnos de P-4 en el aula de inglés. La falta de competencia lingüística previa limitó a algunos alumnos a la hora de desenvolverse en los talleres de inglés.

Desde nuestro punto de vista es fundamental desarrollar competencia lingüística previa a la introducción sistemática de AICLE (Aprendizaje Integrado de Contenidos y Lengua Extranjera), de otro modo esta opción resulta de difícil aplicación. Es necesario contar con una competencia inicial mínima que permita la suficiente fluidez y que no convierta el trabajo en algo tan alejado de las capacidades de nuestro alumnado que genere desmotivación y desinterés (Pérez y Roig, 2004:113). 


\subsubsection{Valoración de la experiencia}

Factores como: la frecuencia de instrucción en inglés, el planteamiento lúdico por lo que respecta a la introducción de la lengua, el uso de recursos atractivos y muy motivadores para los alumnos a la hora de memorizar y reproducir vocabulario y expresiones en inglés, la posibilidad de recrear situaciones comunicativas en inglés de forma natural gracias a la participación de padres de alumnos en el desarrollo de algunas sesiones, etc., han hecho que las sesiones en inglés con los alumnos de P-3 y los talleres con P-5 hayan sido un éxito. Pese a que los resultados conseguidos en los talleres con P-4 no hayan sido tan vistosos, se ha de hablar de una clara satisfacción delante de las demostraciones de pericia comunicativa en lengua inglesa manifestadas por los alumnos a lo largo de todo el curso.

\subsection{Los registros en vídeo}

Los registros realizados en video de algunas sesiones a finales de curso muestran los resultados obtenidos con los alumnos de P-3 como los más exitosos e inesperados de todos. Exitosos porque los tres instrumentos de recogida de datos utilizados en esta investigación así lo demuestran, e inesperados porque a pesar de la garantía de éxito que desde un principio han supuesto: la distribución horaria propuesta para estos alumnos, el planteamiento lúdico de las sesiones y las posibilidades implícitas de aprendizaje de la lengua que supone la instrucción con alumnos tan jóvenes tanto los padres de los alumnos como la maestra no podían esperar que los resultados conseguidos pudieran ser tan alentadores.

Contrastando los datos recogidos a partir del registro de una sesión con los alumnos de P-4, con los obtenidos a partir del diario de campo de la maestra y de los cuestionarios pasados a las familias se puede comprobar que todas las fuentes nos llevan a unas mismas conclusiones. Algo no salió tal y como era de prever ya que los resultados muestran unos índices más bajos de conocimiento y uso del inglés por parte de estos alumnos respecto de sus compañeros. Es decir, a pesar del valor que tiene cualquier planteamiento de propuesta didáctica basada en AICLE, nunca se debe olvidar que esto implica la existencia de una competencia inicial mínima por parte del alumnado que si no se da, favorece la obtención de unos resultados muy similares a los recogidos en la sesión con los alumnos de P-4.

Por lo que respecta a los alumnos de P-5, cabe decir que el registro de una de las sesiones de talleres con este grupo confirma que los alumnos conocen muy bien la dinámica de la sesión, la comparten, participan de ella, la disfrutan y se acaban convirtiendo en los protagonistas absolutos de todas y cada una de las situaciones de comunicación y diálogo que se dan en el aula. Sin lugar a dudas, los resultados conseguidos con los alumnos de P-5 a partir de la introducción del inglés a través de los talleres cubren las expectativas que se plantearon en un principio.

\section{Conclusiones}

A la luz de los resultados obtenidos una vez diseñada, aplicada y evaluada la propuesta aplicada en el CEIP Sant Cristòfor podemos decir que es posible avanzar la introducción de la lengua inglesa en el parvulario obteniendo buenos resultados. 
Cabe, en cualquier caso, procurar favorecer que los alumnos experimenten su primera toma de contacto con la lengua procurando que ésta sea atractiva y que les motive a reproducir pequeños diálogos espontáneos y significativos. Se deben de eliminar las actividades relacionadas con la enseñanza formal de la lengua y dejar de lado metodologías, estrategias y técnicas tradicionales para apoyar nuevas formas de enseñanza y aprendizaje de lenguas extranjeras.

A continuación, se procederá a exponer el grado de cumplimiento de los objetivos propuestos en esta investigación.

Poner los medios teóricos y prácticos necesarios para demostrar que es posible avanzar la enseñanza del inglés L.E. a la Educación Infantil.

La valoración de la aplicación y evaluación pertinentes es muy positiva. A pesar de haber sido necesario realizar algunas modificaciones sobre la programación prevista, el funcionamiento de las diferentes sesiones y la realización de las actividades diseñadas han sido muy satisfactorias y han superado cualquier expectativa inicial.

Elaborar una propuesta didáctica que permita avanzar la enseñanza del inglés en Educación Infantil.

A partir del trabajo de investigación mencionado en el objetivo anterior, se ha elaborado una propuesta didáctica concreta. Esta labor se ha realizado en función de tres factores bien diferenciados:

Los acuerdos tomados por las administraciones en materia de enseñanza de lenguas extranjeras en Educación Infantil.

Las necesidades concretas del centro.

Las necesidades propias de las personas implicadas en el diseño, aplicación y evaluación de la propuesta didáctica.

Aplicación y evaluación de la propuesta didáctica elaborada en un contexto escolar similar al de las escuelas públicas.

La aplicación y evaluación de la propuesta se ha realizado en una escuela que, a pesar de ser pública, disfruta de unos privilegios que la hacen extraordinaria. Este hecho hace prever que la aplicación de esta propuesta en otras escuelas públicas requiera de las revisiones y adecuaciones pertinentes.

Acercar a los alumnos al inglés a través de la enseñanza no formal de la lengua.

Es precisamente la ilusión que demuestran los alumnos delante de esta situación lo que los hace diferentes de los adultos ya que estos últimos, en condiciones similares, se verían ridículos y sentirían vergüenza. En consecuencia, una propuesta de avance de la introducción del inglés en el parvulario no puede ser una copia simplificada de la correspondiente dirigida a alumnos mayores. Más bien, se impone la necesidad de crear nuevas propuestas diseñadas bajo un enfoque centrado en los alumnos de Educación Infantil.

\section{El futuro de la propuesta}

La propuesta presentada conseguirá un éxito mucho más rotundo si cuenta con una continuidad, de características muy similares a las de la propuesta para la Educación Infantil, en la Educación Primaria. 
Una propuesta para la Educación Primaria responsable de continuar la labor hecha al parvulario debería de velar por garantizar que los alumnos se comuniquen en el aula en inglés. Para hacerlo, no se deberían de dejar de lado ninguno de los siguientes aspectos:

- Crear situaciones de necesidad de comunicarse en inglés en el aula.

- Promover la creación de espacios de comunicación significativos para los alumnos.

- Recurrir al uso de materiales reales muy cercanos a los alumnos.

- Hacer uso del juego, la música, la creatividad, la expresión corporal, etc. para garantizar situaciones de diálogo.

- Optar por alternativas a formas de instrucción tradicionales de la lengua inglesa.

- Valorar la posibilidad de eliminar la asignatura de inglés a favor de la enseñanza de otras áreas del currículum en inglés.

- Evitar la limitación de espacios y recursos a la hora de enseñar en inglés.

- Conjugar la introducción de la lecto-escritura en inglés con el desarrollo oral de la lengua que se viene realizando desde el parvulario.

Los resultados conseguidos después de un año de aplicación de la acción didáctica hacen pensar que la perfección y continuidad de la propuesta presentada favorecerán la correcta adquisición del inglés por parte de los alumnos en el futuro. Esto será posible siempre y cuando los profesionales del centro y las autoridades pertinentes consideren la necesidad de ampliar el abanico de actividades para los alumnos de Educación Primaria. Los alumnos que hayan experimentado su primer contacto con el inglés en la Educación Infantil tendrán que tener la oportunidad de poderse beneficiar de esta ventaja en la Educación Primaria. Lo adecuado sería que todos los alumnos pudieran tener una sesión de enseñanza en inglés diaria y que esta frecuencia fuera aumentado a medida que pasa el tiempo. De esta manera nos acercaríamos a una situación de doble inmersión lingüística.

\section{REFERENCIAS BibLIOGRÁFICAS}

Caballero de Rodas, B. y Masats, D. (1999). "Les llengües estrangeres com a vehicle d'aprenentatges escolars", en Perspectiva Escolar, 232: 19-25.

Cantero, F.J. y de Arriba, J. (1997). Psicolingüística del discurso. Barcelona: Ediciones Octaedro.

Català, M. (2001). "Tractament de les llengües i les TIC a l'escola", Material inèdit elaborat per a l'obtenció d'un Pla Estratègic del Departament d'Ensenyament.

Cohen, L. y Manion, L. (1990). Métodos de investigación educativa. Madrid: La Muralla.

Generalitat de Catalunya, Departament d'Ensenyament (2004). La llengua estrangera al cicle inicial. Aspectes organitzatius i didàctics. Barcelona: Servei de difussió i Publicacions.

McKernan (1996). Investigación-acción y currículum. Madrid: Morata.

Muñoz, C. y Nussbaum, L. (1997). "Les enjeux linguistiques dans l'éducation en Espagne", en Muñoz, C., Nussbaum, L. y Pujol, M. (Eds.). Appropriation de langues dans une situation de contact, en AILE, 10:5-20.

Navalón, J.D. (1996). “Aprender a usar el lenguaje en la escuela infantil”, en Aula, 46: 9-13.

Nussbaum, L. (1999).'Perspectives actuals per a l'ensenyament i l'aprenentatge de llengües estrangeres a l'educació obligatòria", en Perspectiva Escolar, 232: 2-8. 
Pedrós Pons, N. (1994). Experiències d'Innovació Educativa. Educació Infantil. 'Barcelona: Departament D'Ensenyament.

Pérez, P. y Roig, V. (2004). Enseñar y aprender inglés en educación infantil y primaria (Vol. I). Barcelona: ICE-Horsori. 